\title{
Modelling of Acoustic Signals from Partial Discharge Activity
}

\author{
Syed Aqeel Ashraf ${ }^{1}$, Brian G. Stewart ${ }^{2}$, Chengke Zhou ${ }^{2}$ and J. Mohd Jahabar ${ }^{1}$ \\ ${ }^{1}$ Faculty of Electrical Engineering, Salalah College of Technology, Salalah-211, OMAN \\ ${ }^{2}$ School of Engineering, Science and Design, Glasgow Caledonian University, Cowcaddens Road, \\ Glasgow, G4 0BA, UK
}

\begin{abstract}
This paper presents an initial simulation study of the propagation of Partial discharge (PD) Acoustic Emission (AE) signals from high voltage equipment. The paper helps to investigate the propagation behaviour of the $\mathrm{AE}$ waves at the interface of two different media namely oil and steel. Reflection, refraction and scattering of the PD shock waves are simulated and displayed. The initial results show that the signals as expected can alter significantly based on the properties of the oil and the steel. The Partial Differential Equations (PDE) describing the signals have been solved using Finite Element Analysis (FEA). Through studying the theoretical aspects of PD AE signals, researchers and practitioners will better understand the properties and characteristic of PD experimental and practical high voltage equipment signatures e.g. power transformers.
\end{abstract}

Index Terms -- Acoustic emission, Finite Element Analysis, Partial Discharges, Partial differential equations, Modelling.

\section{INTRODUCTION}

Partial discharges (PDs) are small surges or sparks of current which occur generally in manufacturing defects in an electrical insulation system when the applied voltage exceeds a specific threshold value. Such undesirable discharges, can over time, deteriorate the insulation and shorten the lifetime of equipment resulting in a potentially catastrophic, costly, and unforeseen failure. Understanding PD and its causes can help diagnose problems and predict the expected lifetime of a dielectric and therefore the HV equipment. For insulation used in high temperature power equipment applications such as cables, transformers, and fault current limiters, PD is thought to be the primary degradation mechanism of the electrical insulation. One of the more common defects in a dielectric insulator is a void that forms as a bubble in the curing of an epoxy dielectric [1].
In recent literatures, many experiments have demonstrated the viability of utilizing $\mathrm{AE}$ signals as caution indicators before any catastrophic failure occurs as a consequence of PD activity [2]-[5]. However, the theoretical aspects of propagation modes related to $\mathrm{AE}$ phenomena in plant items is, surprisingly, still largely unexplored. This gap between theoretical and experimental investigations has therefore prompted a need for developing a mathematical simulation model of AE. Such a model will provide a better understanding of the emission and propagation processes of acoustic PD signals within typical power plant insulation and associated enclosed geometries. This will also assist practitioners in more accurate interpretation of $\mathrm{AE}$ signals and phenomena within these plant items.

\section{PROBLEM FORMULATION}

A PD event can be considered as a small "explosion" or "micro-crack" which leads to the emission of ultrasonic pressure waves. In the first instance, these can be considered as shock waves having a spherical wave front. In reality, the pressure waves are more complex, but for an initial evaluation, the simplest form of signal emission is considered. These signals travel towards sensors through various propagation materials. At boundary surfaces of different media, reflection, refraction, deflection and attenuation occur according to the acoustic impedances of the materials. Following standard acoustic propagation theory, at the PD site, the spherical wave front contains two types of pressure waves i.e. Longitudinal and Shear waves. For Longitudinal waves, the motion of the medium is purely in the direction of propagation. With Shear waves, the motion is transverse to the direction of propagation.

The standard Partial Differential Equation (PDE) that governs the propagation of an acoustic wave within isotropic media is given by:

$$
\partial^{2} P / \partial t^{2}=C^{2} \nabla^{2} P
$$

In equation (1), $P$ is the pressure wave field $(P a), t$ is 
the time $(s)$ and $C$ is the acoustic wave velocity $\left(m s^{-1}\right)$. This equation is a combination of three basic equations, which describe continuity, conservation of momentum, and elasticity of the medium.

As pressure waves contain both Longitudinal and Shear waves, the wave equation can be written as,

$$
\begin{aligned}
& \frac{\partial^{2} L}{\partial t^{2}}=C_{L}^{2} \nabla^{2} L \\
& \frac{\partial^{2} S}{\partial t^{2}}=C_{s}^{2} \nabla^{2} S
\end{aligned}
$$

where $L$ and $S$ are the Longitudinal and Shear pressures, and $C_{L}$ and $C_{S}$ are the velocities of Longitudinal and Shear waves respectively.

The velocity differs for each wave type and can be determined from the density and elastic properties of the material, i.e. from the young modulus $(E)$; density of the medium $(\rho)$; the Poisson ratio $(\mu)$; in solids and the bulk modulus $(K)$, density of the medium $(\rho)$ and the Viscosity $(\eta)$ in liquids. The waves can be initiated through application of appropriate initial conditions, defined through:

$$
\left.L\right|_{t=0},\left.\frac{\partial L}{\partial t}\right|_{t=0},\left.S\right|_{t=0},\left.\frac{\partial S}{\partial t}\right|_{t=0}
$$

The above PDEs can be solved and simulated using Finite Element Methods (FEM).

In the current literatures, to solve the PDEs that describes the wave equation, Finite Difference Time Domain (FDTD) method has been widely used [7]-[11]. However there are some limitations with FDTD technique, e.g.

- It is unable to handle complex geometries and boundaries of different materials.

- To process the problem it needs more computational memory than FEM techniques.

- Is is unable to handle higher order approximations especially multi-dimensional problems.

- The quality of the approximations used between for example mesh/grid points is poor.

In numerical analysis, to overcome the limitations of FDTD, Finte Element Method (FEM) is used for solving PDEs approximately. Solutions are approximated by either eliminating the differential equation completely ( as steady state problems), or rendering the PDEs into equivalent Ordinary Differential Equations, which are then solved using standard techniques such as finite differences, etc. The use of FEM in engineering for the analysis of physical systems is commonly known as Finite Element Analysis (FEA).

In this contribution of work the FEA tool, FEMLAB has been used to solve the PDEs equations (2) and (3). This software is a powerful, interactive environment for modelling and solving scientific and engineering problems based on PDEs. When solving the PDEs that describe a model, FEMLAB applies FEM. It has an extremely broad applicability, and can model a large number of physical phenomena in many disciplines including acoustics, electromagnetics, fluid dynamics, wave propagation and heat transfer.

\section{PROBLEM DEFINITION, SIMULATION RESULTS AND DISCUSSIONS}

The basic simulation is based on two 2-dimensional regions $R_{1}$ and $R_{2}$ of a simple propagation geometry resembling a simple $\mathrm{HV}$ environment with a point source PD. The main objective in the first instance is to demonstrate at a very simple level the propagation characteristics of the $\mathrm{AE}$ in one direction within oil and also within a boundary of steel. $\mathrm{R}_{1}$ is considered as an oil filled steel tank of size $30 \mathrm{~cm}$ by $20 \mathrm{~cm}$ with the thickness of the steel wall presumed to be $0.5 \mathrm{~cm} ; \mathrm{R}_{2}$ is steel of size $5 \mathrm{~cm}$ by $20 \mathrm{~cm}$ and is considered as a boundary at one end of the oil container geometry.

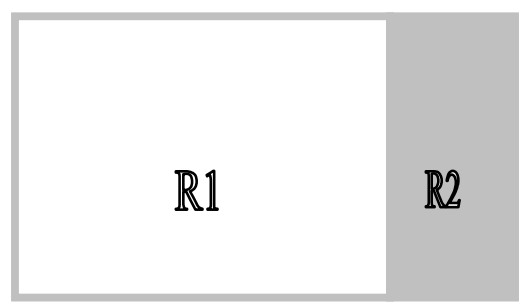

Fig.1 Investigative 2Dgeometry with regions $R_{1}$ and $R_{2}$

There are no internal obstacles considered in this simulation as this is an investigation to illustrate the propagation of simple $\mathrm{AE}$ within this environment. Of course in reality, further investigations will include 3D geometries and also the position of internal obstacles. The PD can be considered as a point source and therefore the "pulse" has a spherical wave front which will be attenuated and reflected according to the acoustic impedances of the oil and steel through which it travels. It is also assumed that the pressure formula for the "explosion" of the PD moves with a spherical front is second order PDE equation (1). 
The oil region $\mathbf{R}_{\mathbf{1}}$ is modelled as Class-I type transformer mineral oil according to the IEC60296 Standard. The temperature is presumed to be $20^{\circ} \mathrm{C}$ with a corresponding density of $854 \mathrm{kgm}^{-3}$ and viscosity 13.0 cSt. The steel region $\mathbf{R}_{\mathbf{2}}$ is modelled as AISI 4340 steel with a Young's modulus $\mathrm{E}$ of $205 \times 10^{9}$, density $\rho$ of $7850 \mathrm{kgm}^{-3}$ and a Poisson's ratio $\mu$ of 0.28 . The initial values should match the boundary conditions to keep the solution well behaved throughout the simulation. If we start at $\mathrm{t}=0$, then the two dimensional pressure can be described through:

$$
P(0)=a \tan \left[\cos \left(\frac{\pi x}{2}\right)\right]
$$

and

$$
\frac{\partial P(0)}{\partial t}=3 \sin (\pi x) e^{\sin \left(\frac{\pi}{2} y\right)}
$$

Where $\mathrm{P}(\mathrm{o})$ is the pressure at time $\mathrm{t}=0$. These are the initial values that satisfy the boundary conditions. The arctan and exponential functions will serve to introduce further modes of propagation into the solution.

To model the 2-Dimensional, $\mathrm{R}_{1}$ oil and $\mathrm{R}_{2}$ steel environments in the FEM, these regions have been divided into different meshes. As the initial conditions have continuous derivatives of all orders we have used a low resolution mesh in order to speed up the calculations.
It consists of 214 nodes and 380 elements and takes around 13 seconds to solve the wave equations. Clearly, the greater number of mesh nodes and elements then better the results.

The PD is modelled as a point source with mathematical form at the centre point of region $R_{1}$. The variation of the pressure of the PD wave, the reflection, refraction and scattering of the shock wave can therefore be modelled and evaluated over time, providing a clear picture of the behaviour and characteristic nature of the waves at any point in the oil or steel and at any time after the PD has occurred. The results are shown in Fig. 2, 3,4 and 5.

In Fig. 2 the acoustic shock wave is propagating in oil as described by the blue colour. In this diagram the shades of blue colour tell us that the wave is propagating towards steel boundary with high pressure. When an acoustic wave propagates from one medium to another which has different acoustic impedances, reflection and refraction will take place. The spherical wave hits the tank wall at the steel boundaries on the $\mathrm{x}$-axis as the blue colour propagates over time. We can see the reflection of the wave as the red colour and its transmission and refraction in steel with light red colour. The colour change is due to attenuation of the wave which results in reduction of the propagated energy.

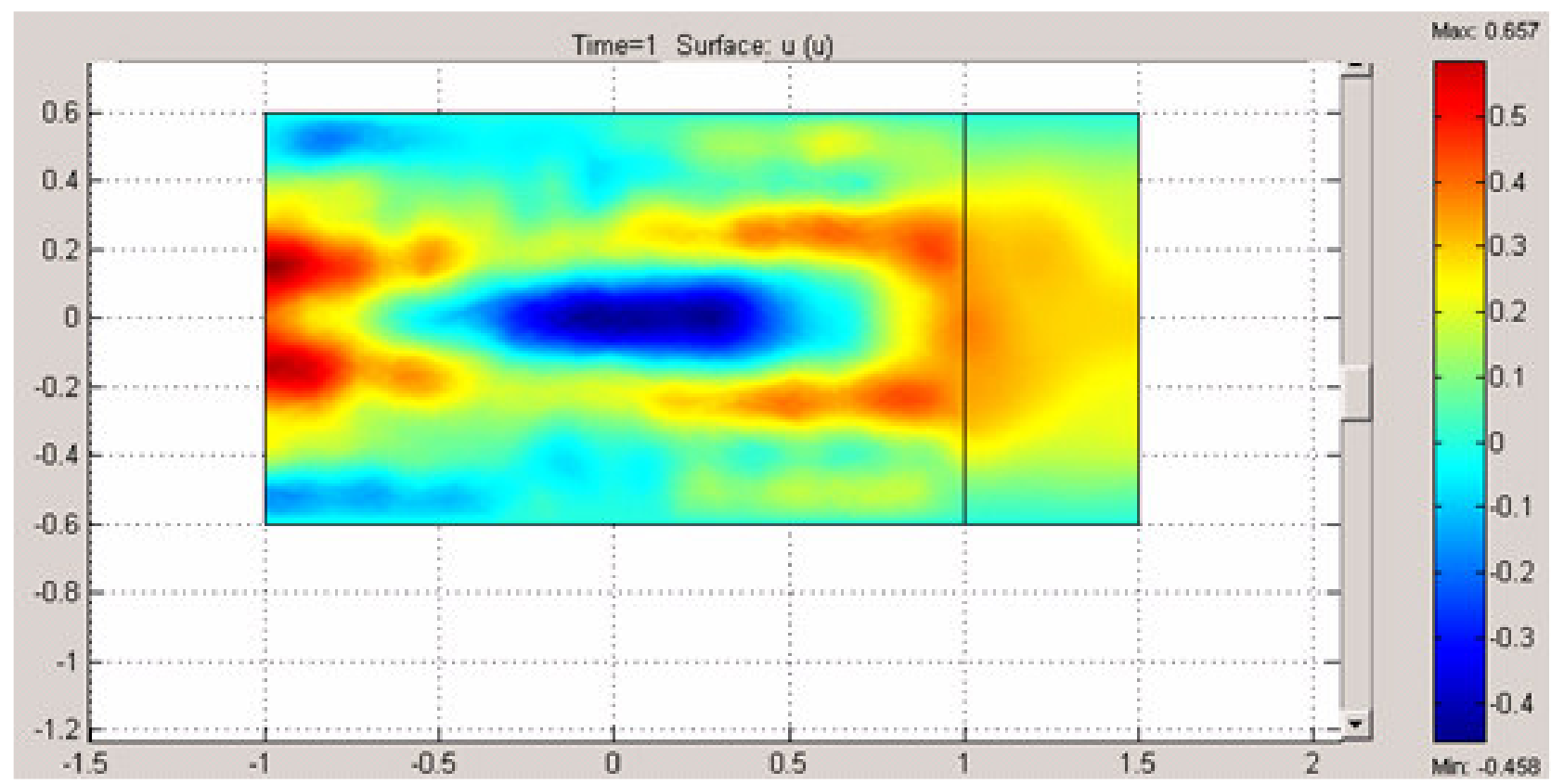

Fig. 2. Propagation of Wave in oil and steel 


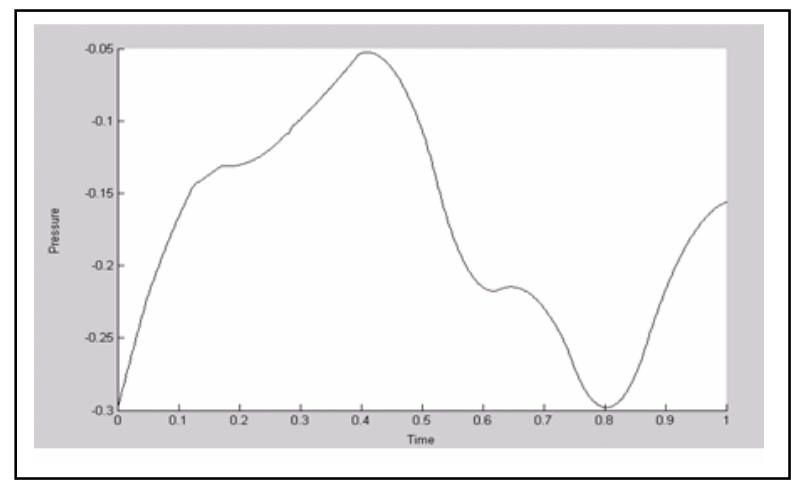

Fig. 3. Variation of pressure of PD with time

In Fig. 3, the pressure (in $\mathrm{Pa}$ ) is evaluated in region $\mathrm{R}_{1}$. The first peak is for PD impulse which arises after 0.4 sec. We know that the Amplitude of the spherical wave is inversely proportional to the radius of wave front. As the shock or spherical wave moves, its radius increases so that the amplitude will decrease. It is clear in fig. 3, that the pressure of the PD decays in due course of time. It seems that the second peak is small in amplitude, which shows the PD acoustic wave amplitude in steel. The small amplitude is due to the big difference in the acoustic impedance of the two media namely oil and steel.

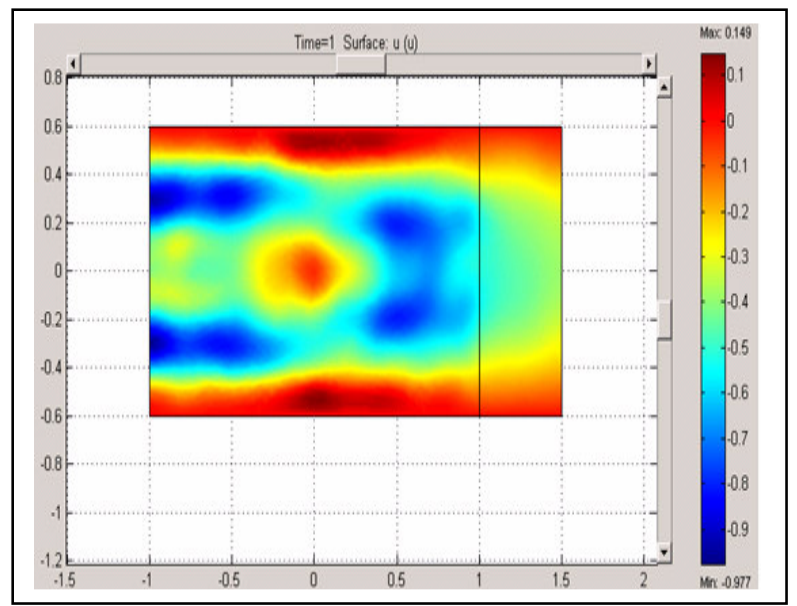

Fig.4. Propagation of wave with rise in temperature

Fig. 4, shows the propagation of acoustic waves when the temperature of the oil is increased due to multiple PDs. More energy is released during multiple discharges, which causes oil to be hot. As this scenario exists the difference in propagation characteristics is important to understand. With increase in temperature the density of the oil reduces and hence the wave front should be large which can be seen as the wave is moving with yellow and light green colour. The wave also hits the steel tank wall $\mathrm{R}_{2}$. Reflection and refraction can be seen with blue and light green colours respectively.

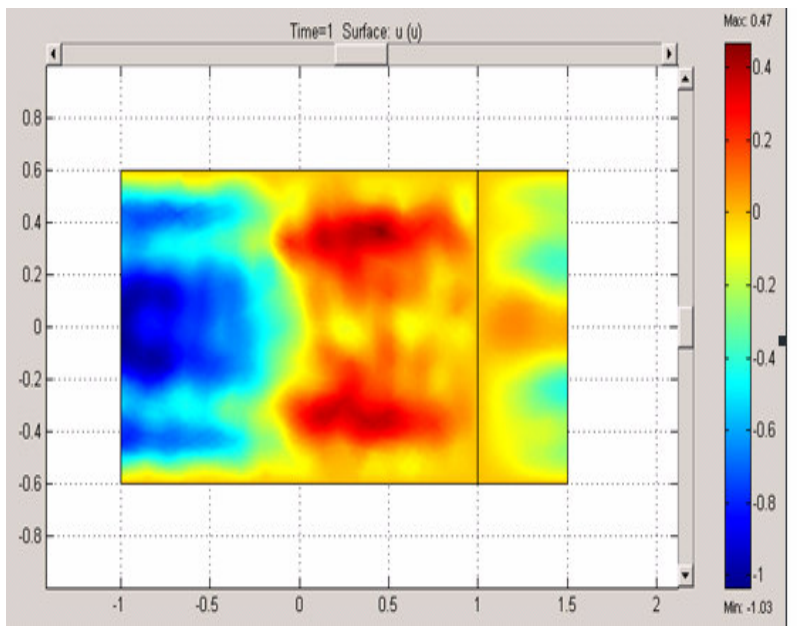

Fig. 5. Reflection, refraction and scattering of shock wave.

In Fig. 5, the scenario in Fig 3 has now been forwarded to time and the shock wave moves in the oil with blue colour. It hits to the steel tank wall $R_{2}$ and is reflected back with red colour. The wave in blue colour shows that the pressure is large. The transmitted or refracted wave can be seen with light red mix with yellow colour. Signal scattering can also be seen with light yellow colour.

\section{CONCLUSIONS}

We have presented a mathematical model for PD acoustic wave in the form of Partial differential Equations. The FEMLAB, a FEA tool, is used to simulate the propagation of the acoustic wave through the oil and steel. Animation results give a clear picture of propagation, attenuation, reflection and refraction of the wave. It is interesting to see that in this simple simulation, the nature of the $\mathrm{AE}$ waves can be quite complex depending on the position of the PD, the reflection of the steel wall. However, perhaps importance of the simulation work will allow the measurement of $\mathrm{AE}$ at the steel tank to be better understood and interpreted more accurately as a function of the steel, the oil, the PD position and the oil temperature. Future work will involve simulating with more complicated 3-D geometries in order to understand better the propagation of acoustic wave through a real transformer core as well as path obstacles. 


\section{ACKNOWLEDGEMENT}

The authors wish to express their sincere thanks to Dr. Ajeet Singh and Dr. Tarig Abdallah for their technical discussions.

\section{REFERENCES}

[1] M.Pompili,C., Bartnikas," Partial Discharge Pulse Sequence Patterns and Cavity development Times in Transformer Oils" IEEE Transactions on Dielectrics and ElectricalInsulation,Vol 12(2),pp.78-94, April 2002

[2] Wadysaw Opydo, "Study of elastic waves of acoustic frequencies generated by surface partial discharges of solid insulators in vacuum", Science Direct Journal, Vol 74(1),pp.85-92, May2004.

[3] Lundgaard, G. Tangen,B., "Acoustic Diagnosis of GIS", IEEE Transactions on Power Delivery, Vol 7, No.1,pp.287-294, Jan.1992

[4] T. Boczar,"Identification of a Specific Type of PD from Acoustic Emission Frequency Spectra" IEEE Transactions on Dielectrics and Electrical Insulation, Vol 8(4), pp.43-51, Aug 2001

[5] B.R.Varlow, D.W.Auckland, "Acoustic Emission analysis of high voltage insulator, "IEE Proc.- Science Measurement. Technology., Vol146 (5), pp.76-84, Sept.1999.

[6] M.M. de A.Olivieri, W. A. Mannheimer,"On the use of Acoustic Signal for Detection and location of Partial
Discharges in Power Transformers," IEEE Int. Symposium on Electrical Insulation, pp.259-262, Apr.2000.

[7] Wong, K.L., Shihab, S., "Radiating Signal Model for Broadband Acoustic Emission from H.V. Equipment" IEEE Transactions on Dielectrics and Electrical Insulation, Vol 2(2),pp.211-218, Feb 2002

[8] Lundgaard, L E,"Partial Discharge- Pt.XIV "Acoustic partial discharge detection - fundamental consideration", IEEE Elect. Insul.Mag., Vol 8, No.4,pp. 25-31, Aug1992

[9] Lundgaard,L E,"Partial Discharge- Pt.XIV, "Acoustic partial discharge detection - practical consideration", IEEE Elect. Insul.Mag., Vol 8, No.5,pp.34-43, December1992

[10] Phung, B.T., Blackburn, "Acoustic Measurement of Partial Discharge Signals", $9^{\text {th }}$ International Symp. On High Voltage Engineering, Australia, Agu.2003

[11] Akumu,A.O.,F.Adachi and K.Arii, "A 3-D Numerical Simulation of Partial Discharge Acoustic Wave Propagation in a Model Transformer",IEEE Int. Symp. On Electrical Insulation, USA, pp183-186, Apr.7-10, 2002

[12] Darley, V.,"Partial Discharges within Power Transformers and the use of ultrasonic techniques in their location,"IEE Conference, pp.231-236, June1990. 